\title{
Abbildungs- und Tabellenverzeichnis
}

Abb. 1: Karte der „Anspruchsgebiete“ verschiedener nationalistischer Lager im östlichen Europa aus: Szczerek, Międzymorze (2017), Einband. — [vor 1]

Abb. 2: „Die erneute Unterwerfung der Rus'“ - Jan Matejko 1888/1889. - 5

Abb. 3: Die Wojewodschaft Ruthenien im 15. Jh. - aus: Janeczek, Authority (2002), 124. — 8

Abb. 4: Objektorte in den Urkunden Kazimierzs III. (1340-1370). - 59

Abb. 5: BCzart, Perg. Nr. 250 (A219); 1397 Sep. 29; Wtadystaw II. Jagiełto bestätigt auf Bitten des Nicolaus Myssnar die Urkunde des Johannes von Tarnów bezüglich der Übertragung des sächsisch-magdeburgischen Rechts für Sokil'nyky. - 475

Abb. 6: AGAD, MK, Lustracje, dz. XVIII, Bd. 51, 32 f.: Liste der von der Familie Herbutów im Zuge der Revision von 1469 vorgelegten Dokumente. -476

Abb. 7: AGAD, tzw. ML, IV.B, Bd. 9, fol. 4v (A547): Eintrag über den Pfandbrief für Semenivka von $1433 \mathrm{im}$ Verzeichnis der Lustration von 1564. -476

Abb. 8: BCzart, Perg. Nr. 565 (B9); 1368 Jan 1; wohl älteste erhaltene Originalurkunde Ottos von Pilica, Capitaneus Russie, über den Kauf von Ščyrec' durch Gan'ko Svarc'. — 477

Abb. 9: ЦДІАУЛ, ф 131, оп. 1, од. 5 (В10); 1368/69; (a) Urkunde Ottos von Pilica, Capitaneus Russie, über den Erwerb eines Hofes in der Nähe von L'viv durch Vjatslav Dmitrov'skij. — 477

Abb. 10: ANKr, Oddział III, Perg. Nr. 861 (A81); 1375 Dez. 25/31; Wtadystaw Opolczyk verleiht dem Danilo Dažbogovyč die Orte Rožnjativ, Sychiv u. a. - 478

Abb. 11: ANKr, Oddział III, Perg. Nr. 861 (A81); 1375 Dez. 25/31; Majestätssiegel Władysław Opolczyks (rechts Gegensiegel). -478

Abb. 12a: AGAD, Perg. Nr. 4680 (A283); 1408 Nov. 28; Majestätssiegel Wtadystaws II. Jagietto. -479

Abb. 12b: AGAD, Perg. Nr. 4709 (A536); 1433 Juni 24; Mittleres Wappensiegel Wtadystaws II. Jagietło. -479

Abb. 12c: AGAD, Perg. Nr. 4688 (A357); 1421 Nov. 20; Kleines Wappensiegel Wtadystaws II. Jagiełto. -479

Abb. 13: Niewodniczański, Signatur A 27 (B25); 1388 Apr. 15 (?);Johannes von Tarnów, Capitaneus Russie, bestätigt den Verkauf von Krościenko durch Hleb Dvorskovyč an Petrus, Sohn des Andreas. -480

Abb. 14a: BCzart, Perg. Nr. 272; 1404 Okt. 19; ein Gericht unter dem Vorsitz der beiden Bischöfe von Przemyśl, lateinischer und orthodoxer Konfession; Pergamentbogen mit Urkundentext in lateinischer Sprache. - 481

Abb. 14b: Pergamentbogen mit Urkundentext in ruthenischer Sprache. - 481

Abb. 14c: BCzart, Perg. Nr. 272; 1404 Okt. 19; Siegel des Matthäus, lat. Bischof von Przemyśl (links); Siegel des Athanasius, orth. Eparch von Przemyśl. (rechts). — 482

Abb. 14d: BCzart, Perg. Nr. 272; 1404 Okt. 19Befestigung der Siegel an beiden Pergamentbögen. -482

Abb. 15: AGAD, Perg. Nr. 5771 (A374); 1423 Juni 10; Władystaw II. Jagiełto verpfändet dem Nicolaus Füllstein das Dorf Kolpecz; unten rechts: Relationsvermerk durch Fiodoras Liubartaitis. -483

Abb. 16: AGAD, Perg. Nr. 6559 (A291); 1410 Dez. 20; Wtadystaw II. Jagiełto verpfändet dem Petrus Kromidowski das Dorf Wacowice. -483

Abb. 17a: ЦДІАУЛ, ф. 131, оп. 1, од. 39; 1407 Sep. 26 (recto); Nicolaus, Dekan des Bistums Przemyśl, lädt im Streitfall zwischen der Stadt L'viv und Johannes Ruthenus, Pfarrer der Marienkirche von L'viv, letzteren vor sein Gericht und transsumiert die Urkunden des Bischofs von Kraków sowie der Bulle Gregors XI. in dieser Sache. -484 
Abb. 17b: ЦДІАУЛ, ф. 131, оп. 1, од. 39 (verso); Notarielle Bestätigungen, dass die Urkunde in L'viv (sowohl vor dem Erzbischof als auch in der Marienkirche) und in Kraków verlesen wurde. -485

Abb. 17c: ЦДІАУЛ, ф. 131, оп. 1, од. 39; Siegelreihe. - 486

Abb. 18: AGAD, MK 54, fol. 232v; Registereintrag in den polnischen Kronmatrikeln von 1537 Sep. 13: Beginn des Transsumpt der Urkunde von Wtadysław II. Jagiełło aus dem Jahr 1416, AGZ III, Nr. 87 (A313). -486

Abb. 19: ЦДІАУЛ, ф. 9, оп. 1, спр. 66, 153; Eintrag ins Burggericht von L'viv aus dem Jahr 1573 mit den Transsumpten Kazimierz IV. von 1467 (oben) und Wtadystaw II. Jagietto von 1425 (unten, vgl. A422) bezüglich der Lokation von Hlynjany durch Johannes von Tarnów, Capitaneus Russie, im Jahr 1397 (B 40). -487

Abb. 20: ЦДІАУЛ, ф. 9, оп. 1, спр. 568, 2422 f.; Eintrag ins Burggerichtsbuch von L'viv aus dem Jahr 1754 allen sechs Transumpten bezüglich der Lokation von Hlynjany. -488

Abb. 21: ЦДІАУЛ, Ф. 9, оп. 1, спр. 568, 2424 f.; Eintrag ins Burggericht von L'viv aus dem Jahr 1754 allen sechs Transumpten bezüglich der Lokation von Hlynjany. -488

Schaubild 1: Überlieferungsstränge der Lokation von Ščyrec'. — 33

Schaubild 2: Aufenthaltszeiten Wtadystaw Opolczyks in Kronruthenien nach Jahren. - 102

Schaubild 3: Die in AGZ IV, Nr. 11 enthaltenen Urkunden zum Jahr 1406. - 212

Schaubild 4: Die in AGZ IV, Nr. 15 enthaltenen Urkunden zum Jahr 1407. - 214

Schaubild 5: Querschnitt herausgehobener Akteure in Kronruthenien (1340-1434). -260

Diagramm 1: Häufigkeit kopialer Überlieferungsträger. -29

Diagramm 2: Verteilung der kopialen Überlieferungen (14.-18. Jh.). - 34

Diagramm 3: Zeitliche Verteilung der urkundlichen Überlieferung 1340-1434. -43

Diagramm 4: Inhaltliche und zeitliche Verteilung der Urkunden Kazimierzs III. (1340-1370). — 58

Diagramm 5: Gegenüberstellung der Personenkreise in den Urkunden Kazimierzs III. und Ottos von Pilica, Capitaneus Russie, 1349-1370. - 74

Diagramm 6: Inhaltliche und zeitliche Verteilung der Urkunden Władysław Opolczyks (13711386). -88

Diagramm 7: Auftritte als Empfänger oder Zeuge in den Urkunden Wtadystaw Opolczyks. —- 104

Diagramm 8: Inhaltliche und zeitliche Verteilung der Urkunden Władysław II. Jagiettos

(1390-1398). - 125

Diagramm 9: Anwesenheit Władystaw II. Jagiełtos nach Tagen pro Jahr (1390-1398) - 126

Diagramm 10: Durchschnittliche Distanz in Kilometern zwischen Ausstellungsort und Objektort nach Empfängergruppen sortiert (1390-1399). - 132

Diagramm 11: Empfänger und Zeugen in den Urkunden Wtadysławs II. und des Capitaneus Russie (1387-1393). - 137

Diagramm 12: Inhaltliche und zeitliche Verteilung der Urkunden Wtadystaw II. Jagiettos (1399-1409). - 147

Diagramm 13: Anwesenheit Wtadystaw II. Jagiettos nach Tagen pro Jahr (1393-1409). — 147

Diagramm 14: Durchschnittliche Distanz zwischen Objektort und Ausstellungsort nach Empfängergruppen in Kilometern (1399-1409). — 159

Diagramm 15: Empfänger und Zeugen in den Urkunden Wtadystaws II. und des Capitaneus Russie (1394-1411). - 170

Diagramm 16: Inhaltliche und zeitliche Verteilung der Urkunden Władystaw II. Jagiełtos (14101423). -173

Diagramm 17: Länge der Aufenthalte Wtadystaw II. Jagiettos in Tagen (1410 und 1423). — 174 
Diagramm 18: Durchschnittliche Distanz zwischen Objektort und Ausstellungsort nach Empfängergruppen in Kilometern (1409-1423). - 191

Diagramm 19: Inhaltliche u. zeitliche Verteilung der Urkunden Wtadystaw II. Jagiettos (1424-1434). - 222

Diagramm 20: Länge der Aufenthalte Władystaw II. Jagiettos in Tagen (1424-1434). — 223

Diagramm 21: Durchschnittliche Distanz zwischen Objektort und Ausstellungsort nach Empfängergruppen in Kilometern (1424-1434). — 245

Diagramm 22: Empfänger und Zeugen in den Urkunden Wtadystaws II. und des Capitaneus Russie (1411-1434). -251

Stammbaum 1: Die Fürsten von Halyč-Volyn' im Geflecht des östlichen Europas. 489

Stammbaum 2: Der Kočak-Klan, Zweig Ivanonyč. - 490

Stammbaum 3: Der Kočak-Klan, Zweig Lojevyč, bzw. Davydiv/Klusowie. - 490

Stammbäume 4 und 5: Der Kočak-Klan, Zweig Bybel'skyj (links) bzw. Zweig Dvorskovyč (rechts). -491

Stammbäume 6 und 7: Der Kočak-Klan, Zweig Slonečkovyč (links); Der Klan Kuzmič (rechts). -491

Stammbäume 8 und 9: Familie Teptukovyč (links) und Familie Kadolfovyč (rechts). 491

Stammbaum 10: Famili Kaplyč. - 492

Stammbaum 11: Famili Kmitowie. - 492

Stammbaum 12: Familie Sprowski. - 492

Stammbaum 13: Familien Pileccy/Melsztyńscy/Tarnowscy. — 493

Stammbaum 14: Familien Balowie. -493

Tab. 1: Übersicht der 1469 durch Jacobus Füllstein vorgelegten Urkunden. - 36

Tab. 2: Die Inhaber des Amtes des Capitaneus Russie im ungarischen Regnum Russie (1379-1387). - 110

Tab. 3: Mehrfachauftritte regionaler Zeugen in Urkunden Wtadystaw Jagiettos (13991409). - 161

Tab. 4: Urkunden mit mehreren regionalen Zeugen (1399-1409). - 162

Tab. 5: Übersicht der Erzbischöfe von Halyč/L'viv und Gründungsdokumente des Erzbistums. - 199

Tab. 6: Dokumente zu im Exkurs 2 behandelten Konfliktlinien in chronologischer Reihenfolge. -202

Tab. 7: Übersicht der Bestätigungen vorgeblicher Urkunden der Fürsten von Halyč-Volyn' im Untersuchungszeitraum. -266

Tab. 8: Übersicht der Inhaber des Amtes Capitaneus Russie unter polnischer Herrschaft (1340-1439). - 274 
